\title{
Self-rated health among youth and its associations with physical activity outside school, body mass index and television time in an urban municipality of Portugal
}

\author{
Saúde autorrelatada entre jovens e suas associações com a prática de \\ atividade física fora da escola, índice de massa corporal e tempo de tela \\ em um município urbano de Portugal
}

Jorge Mota', Michael Duncan², Mauro Barros³, José Cazuza de Farias Júnior, José Carlos Ribeiro', Joana Carvalho', Maria Paula Santos ${ }^{1}$, Andreia Pizarro'

\begin{abstract}
This study examined associations of physical activity (PA) outside school, Body mass index (BMI) and television time (TVT) with Self-rated health (SRH) among youth. A total of 1,121 adolescents aged from 10-18 years old participated in this cross-sectional study. PA was obtained through a questionnaire. TVT was accessed by asking the participant how many hours/day did they watch TV in the preceding week. BMI [weight $(\mathrm{kg}) /$ height $^{2}(\mathrm{~m})$ ] were used as an indicator of children's nutritional status. Associations of self-reported PA, TVT and BMI to SRH were assessed by gender, using Chi Squared and multivariate logistic regression controlling for mothers' education level. High levels of physical activity (boys: $\mathrm{OR}=2.43$; $95 \% \mathrm{CI}: 1.24$ to 4.78 ; girls: $\mathrm{OR}=2.02$; $95 \% \mathrm{CI}: 1.26$ to 3.24) were significantly associated with better SRH in adolescents. Obese/overweight boys were less likely to report positive $\mathrm{SRH}(\mathrm{OR}=0.41$; 95\%CI: 0.20 to 0.81$)$ compared to their normal-weight peers. TVT in both genders and being overweight/ obese in girls was not significantly associated with SRH. Results suggest that positive $\mathrm{SRH}$ is associated with PA levels, in both boys and girls, and with better nutritional status in boys. Keywords
\end{abstract}

Nutritional Status; Health Status; Motor Activity; Overweight; Public Health.

\section{Resumo}

Este estudo analisou as associações entre a autoavaliação da saúde (AAS) com a atividade física ( $A F$ ) fora do contexto escolar, o indice de massa corporal (IMC) e o tempo de televisão (TTV). Um total de 1.121 adolescentes de 10 a 18 anos de idade participaram deste estudo transversal. A AF foi mensurada por questionário. OTTV foi obtido pela questão "Na última semana quantas horas por dia você passou assistindo TV?". O IMC foi utilizado como indicador do estado nutricional. As associações entre a AAS e as restantes variáveis foram determinadas, separadamente por género, pelo Chi-quadrado e regressão logística multivariada, controlando para a escolaridade da mãe. Niveis elevados de AF foram associados a uma melhor AAS (rapazes: $O R=2,43$; IC95\%: 1,24-4,78; meninas: $O R$ = 2,02; IC95\%: 1,26-3,24). Rapazes com sobrepeso/obesidade apresentaram menor chance de autoavaliarem o estado de saúde de forma positiva $(O R=0,41$; IC95\%: 0,20-0,81) quando comparados aos de peso corporal normal. O TTV não se associou à AAS em rapazes nem em meninas. Ter sobrepeso/obesidade não se mostrou significativamente associado a AAS em meninas. Os resultados do nosso estudo sugerem que maiores níveis de AF em rapazes e em meninas e menor IMC nos rapazes se associam a uma melhor AAS.

\section{Palavras-chave}

Estado nutricional; Nivel de saúde; Atividade motora; Excesso de peso corporal; Saúde pública.

\section{Introduction}

The health benefits of physical activity (PA) are widely established ${ }^{3}$ and sedentary behavior (SB), such as television time, has been associated with increased risk of several cardiovascular and metabolic diseases in youth ${ }^{4}$. In addition, the prevalence of paediatric obesity has increased ${ }^{5}$ and higher rates of overweight/ 
obesity have been described in Portuguese youth ${ }^{6}$ with scores of $23.1 \% / 9.6 \%$ in girls and $20.4 / 10.3 \%$ in boys, respectively. While all these factors were associated with several biomedical outcomes in youth, the association with perceptions of overall health are scarce. Self-rated health (SRH) is a common measure of health-related quality of life in adults and it was suggested as an overall health indicator among adolescents ${ }^{7}$. SRH has been shown to be a relatively stable construct during adolescence ${ }^{8}$ and is associated with adolescents' overall sense of functioning as well as health-related quality of life ${ }^{9}$. Adolescent SRH are framed not only by their physical health status, but also by personal, socio-environmental, behavioral and psychological factors ${ }^{10}$. Moreover, youth usually use health behaviors (e.g. diet, exercise, alcohol, tobacco) as reference when providing global health status assessments ${ }^{11}$.

So far, the few studies exploring associations between SRH and health behaviors provide mixed results and most of the studies look at health behaviors in isolation despite research suggests that a socio-ecological approach may be more effective in health promotion. It seems then important to examine the relationships among health behaviors in relation to SRH instead of targeting individual behaviors.

There are limited data addressing the association of SRH with PA, nutritional status and Television viewing like a marker of SB in an adolescent population. Furthermore, it is less clear whether any associations between these variables might differ between genders. Examining this issue may be important as during adolescence SRH deteriorates, especially among girls, and a large number of adolescents report subjective health complaints and health concerns ${ }^{12}$. Understanding the degree to which a group of behaviours, namely, PA, SB and nutritional status relate to health may be useful in better structuring public health programmes and initiatives aimed at increasing perceived and real health in adolescents.

Therefore, this study examined associations of physical activity (PA) outside school, Body mass index (BMI) and television time (TVT) with Self-rated health (SRH) among portuguese youth.

\section{Methods}

The present study is a secondary analysis from a broader research designed to study the effect of physical activity and sedentary behaviour in BMI of adolescents from Aveiro. Aveiro is a city and a municipality in Portugal with a total area of $199.9 \mathrm{~km}^{2}$ and a total population of 73559 inhabitants. It is the second most populous city in central Portugal.

Cross-sectional data was collected in 2010-2011 in all 8 public secondary schools from Ilhavo, an urban municipality, in Aveiro District of Portugal. The required sample size was calculated à priori with G.power considering the following parameters: medium effect size of 0.15 ; power of $90 \%$; an error of 0.05 and six predictors namely age, gender, socio economic status. Based on these criteria, the required minimum sample size was 123 students. The initial sample size was increased by $25 \%$ to which $25 \%$ was added to cover possible losses and refusals, therefore final sample size was established as 154 students. However, due to a specific request of the schools to involve all the students in this health research, all the students registered in $7^{\text {th }}$ until $12^{\text {th }}$ grade were invited to take part in the study. All assessments were carried out during a mandatory physical education class. Adolescents that participated in this study were free of medical treatment. A letter informing families that students would be measured was sent home two weeks before measurements took place. Schools approved the study protocol and all parents signed an informed consent form. The same group of researchers car- 
ried out all measures. This study was conducted according to the guidelines of the Helsinki Declaration of Human Studies and was approved by the Portuguese Foundation for Science and Technology and by the Scientific Board of Physical Activity and Health $\mathrm{PhD}$ program.

Anthropometric Measures were taken with the adolescent lightly dressed, in bare or stocking feet. Body height was measured to the nearest millimeter standing upright against a Holtain stadiometer. Weight was measured to the nearest $0.10 \mathrm{~kg}$, using an electronic weight scale (Seca 708 portable digital beam scale). Nutritional Status was estimated by the body mass index (BMI), calculated as the ratio of weight (in $\mathrm{kg}$ ) to height (in $\mathrm{m}^{2}$ ). BMI cut offs for child overweight and obesity cover the age range 2-18 years and are calculated and organized based on the adult cut offs of 25 and 30 at 18 years using linear interpolation between the cut-off points according to age and sex, as described by Cole et al. ${ }^{2}$. Thus, for the purpose of this study nutritional status was defined for two categories: Normal weight and Overweight/Obesity.

Television time (TVT) was obtained through a questionnaire asking participants how many hours/day they usually watched television in the week preceding the examination, including weekends. Adolescents were, then, assigned as one of two groups: the low television time (LTV), and high television time (HTV) users, based upon them reported to spend $\leq 2 \mathrm{~h} /$ day vs. $>2 \mathrm{~h} /$ day watching $\mathrm{TV}$, respectively. In this study, two-week intraclass test-retest correlation coefficients - ICC $(\mathrm{n}=100)$ for this item ranged from 0.78 to 0.94 .

Physical Activity (PA) was assessed by a group of questions developed by Telama et al. ${ }^{1}$ about the weekly frequency of structured and unstructured PA and PA competitions outside school. The questionnaire comprises five questions with four choices per question: (a) Outside school do you take part in organized sport?; (b) Outside school do you take part in non-organized sport?; (c) Outside school, how many times a week do you take part in sport or physical activity for at least 20 minutes?; (d) Outside school hours, how many hours a week do you usually take part in physical activity so much that you get out of breath or sweat?; (e) Do you take part in competitive sport? Overall a maximum of 20 points can be reached with higher scores indicative of higher PA. A PA index was obtained, which divided the sample into four different activity categories, according to the total sum of the points: the sedentary group [0-5]; low active group [6-10]; moderately active group [11-15] and vigorously active group [16-20]. As health-related PA guidelines for youth stress the engagement into moderate-to-vigorous PA, for statistical analysis the subjects were grouped into two categories: the non-active group (NPA) comprising sedentary and low active youth; and the active group (APA) comprising the moderately and vigorously active youth on the basis of their reported physical activity ${ }^{13}$.

Self-rated-health (SRH) was assessed by the question: "In general, how would you rate your health?" Items were scored on a Likert scale with 1 = "poor" to 5 ="excellent". Following the general procedure applied in this study, the no risk vs. risk approach positive vs. negative, respectively was used. This measure was dichotomized into "positive SRH" (excellent, very good and good answers) and "negative SRH" (fair and poor). The two-week test-retest reliability score was $r=$ 0.92. The use of a single-item measurement to assess SRH is consistent with most research involving this indicator of overall health status in adolescents ${ }^{12}$.

Mother's educational level was used as a proxy for socioeconomic level. Information was collapsed into three categories according to Portuguese Educational System: $\leq 6$ years (low), $7-12$ years (medium) and $>12$ (high). 
For statistical analyses means and standard deviations were calculated to describe participants' characteristics. Descriptive statistics included the nutritional status (normal-weight, overweight/obese), PA categories (NPA vs. APA) and TVT $(\leq 2 \mathrm{~h} /$ day vs. $>2 \mathrm{~h} /$ day) frequencies according to SRH groups. The chi-square test $\left(c^{2}\right)$ was used to determine the differences in the proportion of nutritional status (BMI), PA and TVT according to SRH. A logistic regression analysis was performed to assess Odds Ratio (OR) and 95\% confidence intervals (CI) for SRH (as dependent variable) with BMI, PA and TVT (as independent variables), separately. The final model of the multivariate logistic regression analysis looking at the same variables was adjusted for maternal education level as a proxy indicator of socio-economic position (SES). Analyses were run separated by gender as literature reveals gender inequalities in $\mathrm{SRH}^{14}$. Statistical analysis was performed using SPSS 21 software (SPSS Inc., Chicago, IL, USA). The level of significance was set at $\mathrm{p} \leq 0.05$.

\section{Results}

A response rate of $94 \%(n=1,175)$ was obtained from a potential sample of 1,250 adolescents. After eliminating subjects with a majority of missing information the final sample comprised 599 (53\%) adolescent boys and 522 (47\%) adolescent girls aged between 13 and 20 years old $(15.4 \pm 1.9$ years old).

Overall (Table 1), $14 \%$ of girls and $8 \%$ of boys reported a negative $\mathrm{SRH}, 22.2 \%$ of girls and $18.4 \%$ of boys were classified as overweight/obese. About $60 \%$ of girls and $78 \%$ of boys were classified as physically active, while $40.2 \%$ of girls and around $35 \%$ of boys exceeded screen time guidelines of $2 \mathrm{~h} /$ day.

TABLE 1 - Main characteristics of the 1,121 participants. Ilhavo, Portugal, 2010/2011.

\begin{tabular}{|c|c|c|c|c|}
\hline & \multicolumn{2}{|c|}{ Girls $(n=599)$} & \multicolumn{2}{|c|}{ Boys $(n=522)$} \\
\hline & \multicolumn{2}{|c|}{ mean $\pm S D$} & \multicolumn{2}{|c|}{ mean $\pm S D$} \\
\hline Age (years) & \multicolumn{2}{|c|}{$14.9 \pm 1.4$} & \multicolumn{2}{|c|}{$15.0 \pm 1.4$} \\
\hline Weight (kg) & \multicolumn{2}{|c|}{$55.5 \pm 11.6$} & \multicolumn{2}{|c|}{$59.7 \pm 13.2$} \\
\hline Height (cm) & \multicolumn{2}{|c|}{$159.9 \pm 6.1$} & \multicolumn{2}{|c|}{$168.4 \pm 9.6$} \\
\hline $\mathrm{BMI}\left(\mathrm{kg} / \mathrm{h}^{2}\right)$ & \multicolumn{2}{|c|}{$21.6 \pm 3.9$} & \multicolumn{2}{|c|}{$21.7 \pm 3.5$} \\
\hline ESE & $(\%)$ & $95 \% \mathrm{Cl}$ & (\%) & $95 \% \mathrm{Cl}$ \\
\hline Low & 36.0 & $32.3-40.0$ & 37.7 & $33.7-42.0$ \\
\hline Medium & 52.8 & $48.7-56.7$ & 48.9 & $44.6-53.1$ \\
\hline High & 11.2 & 8.9-13.9 & 13.4 & $10.7-16.6$ \\
\hline \multicolumn{5}{|l|}{ SHR } \\
\hline Negative & 14.0 & $11.5-17.0$ & 7.9 & $5.8-10.5$ \\
\hline Positive & 86.0 & $83.0-88.5$ & 92.1 & $89.5-94.2$ \\
\hline \multicolumn{5}{|l|}{ BMI } \\
\hline Normal & 77.8 & 74.3-80.9 & 81.6 & 78.1-84.7 \\
\hline Overweight /obese & 22.2 & $19.1-25.7$ & 18.4 & $15.3-21.9$ \\
\hline \multicolumn{5}{|l|}{ PA } \\
\hline NPA & 40.1 & $36.2-44.0$ & 22.0 & $18.7-26.1$ \\
\hline APA & 59.9 & $56.0-63.8$ & 78.0 & $74.2-81.3$ \\
\hline \multicolumn{5}{|l|}{ TVT } \\
\hline$\leq 2 \mathrm{~h} /$ day & 59.8 & $55.8-63.6$ & 65.3 & $61.1-69.3$ \\
\hline$>2$ h/day & 40.2 & $36.4-44.2$ & 34.7 & $30.7-38.9$ \\
\hline
\end{tabular}

SD: standard deviation; Kg: kilograms; $\mathrm{cm}$ : centimeter; kg/h²: kilogram per square of height; SHR: Self-rated health; 95\% Cl: Confidence interval of 95\%; BMl: body mass index; PA: Physical Activity; NPA: Non active group; APA: Active group; TVT: television time; h/day: hours per day. 
The regression analysis, for boys and girls, respectively are shown in Table 2 and 3. High levels of physical activity (boys: $\mathrm{OR}=2.43$; $95 \% \mathrm{CI}: 1.24$ to 4.78 ; girls: $\mathrm{OR}=$ 2.02; 95\% CI: 1.26 to 3.24) were significantly associated with better SRH in adolescents. Obese/overweight boys were more likely to report negative $\mathrm{SRH}(\mathrm{OR}=0.41$; $95 \%$ CI: 0.20 to 0.81 ) compared to their normal-weight peers. TVT in both genders and across nutritional status in girls was not significantly associated with SRH.

TABLE 2 - Binary logistic regression for the association between level of PA, nutritional status (BMI) and TVT time and SRH in boys. Ilhavo, Portugal, 2010/2011.

\begin{tabular}{|c|c|c|c|c|}
\hline & $\begin{array}{l}\text { Crude analyses } \\
\text { Odds ratio }(95 \% \mathrm{Cl})\end{array}$ & $P$ value & $\begin{array}{l}\text { Adjusted analyses* } \\
\text { Odds ratio }(95 \% \mathrm{Cl})\end{array}$ & P value \\
\hline \multicolumn{5}{|l|}{ Nutricional status } \\
\hline Normal weight & 1 & & 1 & \\
\hline Overweight/obese & $0.49(0.14-0.89)$ & 0.01 & $0.41(0.20-0.81)$ & 0.01 \\
\hline \multicolumn{5}{|l|}{ Physical activity } \\
\hline NPA & 1 & & 1 & \\
\hline APA & $2.44(1.24-4.78)$ & 0.00 & $2.43(1.24-4.78)$ & 0.01 \\
\hline \multicolumn{5}{|l|}{ Sedentary behaviour } \\
\hline$>2 \mathrm{hTVT}$ & 1 & & 1 & \\
\hline$\leq 2 \mathrm{hTVT}$ & $1.01(0.51-1.97)$ & 0.99 & $1.01(0.51-1.98)$ & 0.99 \\
\hline
\end{tabular}

*Adjusted for SES (mother's education level). PA: physical activity; BMI: Body mass index; TTV: television time; SRH: Self-rated health; C195\%: Confidence interval of 95\%; NPA: Non active group; APA: Active group; SES: socio-economic status. P value: significance level.

TABLE 3 - Binary logistic regression for the association between level of PA, nutritional status (BMI) and TVT time and SRH in girls. Ilhavo, Portugal, 2010/2011.

\begin{tabular}{|c|c|c|c|c|}
\hline & $\begin{array}{l}\text { Crude analyses } \\
\text { Odds ratio }(95 \% \mathrm{Cl})\end{array}$ & $P$ value & $\begin{array}{l}\text { Adjusted analyses* } \\
\text { Odds ratio }(95 \% \mathrm{Cl})\end{array}$ & $P$ value \\
\hline \multicolumn{5}{|l|}{ Nutricional status } \\
\hline Normal weight & 1 & & 1 & \\
\hline Overweight/obese & $1.30(0.72-2.31)$ & 0.39 & $1.28(0.70-2.35)$ & 0.42 \\
\hline \multicolumn{5}{|l|}{ Physical activity } \\
\hline NPA & 1 & & 1 & \\
\hline APA & $2.12(1.35-3.38)$ & 0.00 & $2.02(1.26-3.24)$ & 0.00 \\
\hline \multicolumn{5}{|l|}{ Sedentary behaviour } \\
\hline$>2 \mathrm{hTVT}$ & 1 & & 1 & \\
\hline$\leq 2 \mathrm{hTVT}$ & $0.78(0.49-1.25)$ & 0.31 & $0.82(0.51-1.31)$ & 0.40 \\
\hline
\end{tabular}

*Adjusted for SES (mother's education level). PA: physical activity; BMI: Body mass index; TTV: television time; SRH: Self-rated health; C195\%: Confidence interval of 95\%; NPA: Non active group; APA: Active group; SES: socio-economic status. P value: significance level.

\section{Discussion}

Our data showed that among girls 14\% reported negative SRH, with this figure being $7 \%$ for boys. These percentages are lower than those previously reported for European and North American healthy adolescent samples ${ }^{14}$. Portuguese data on the 2012 Health Behavior in School-Age Children (HBSC) report, showed somewhat similar levels of negative SRH in adolescents. According to HBSC report $15 \%$ and $9.5 \%$ of the $13-15 y$ years old girls and boys, respectively, reported negative $\mathrm{SRH}^{14}$. Gender differences are consistent with previous research patterns with 
higher levels of poorer health being reported by adolescent girls when compared to boys ${ }^{15}$. In general, boys seem to feel better in regard to their own health as compared to girls ${ }^{14}$. Indeed, the results of this study indicated a robust pattern of increasing gender differences across age, with 15 -year-old girls as a group at increased risk for health complaints across all countries ${ }^{14}$. Differences in SRH have also been suggested to relate to girls' higher levels of stress associated with achievements ${ }^{16}$, sadness and anxiety ${ }^{17}$. Girls report lower levels of self-esteem and pay more attention to appearance, weight ${ }^{15}$ and social relationships ${ }^{16}$. Furthermore, it has been suggested that having a chronic condition significantly lowered the odds that both younger and older teens would report very good/excellent health ${ }^{15}$.

Health-related behaviors such as physical activity (PA) may play an important role in SRH as youth frequently use health behaviors such as exercise and physical cues as referent when providing health status assessments ${ }^{11,18}$. Moreover, as PA has a relevant role in determining body shape, self-esteem ${ }^{19}$ and physical self-perceptions ${ }^{20}$ one may speculate about its importance for SRH. Also PA is associated with other aspects of SRH such as positive mental outcomes ${ }^{21}$ and higher social interaction $^{22}$. In fact, some previous studies have shown that PA is positively associated with adolescents' self-rated health ${ }^{8}$. Our data show that those who were classified as active were around twice as much more likely to report positive SRH compared to their less active counterparts. Therefore, this data are in line with previously published data pointing-out that SRH among adolescents may differ according to sex and a person's overall level of PA, suggesting that the association is stronger among boys and among more active adolescents ${ }^{23}$. In addition, Page and Suwanteerangkul ${ }^{24}$ concluded that Thai adolescents who rated themselves as "very healthy" were more likely to engage in vigorous physical and muscle-strengthening activities, play on sports teams, and score higher on the physical activity index compared to adolescents who rated themselves as "healthy" or "not healthy". Although the variability is great, young people classified as fit have tended to be more active. As an example it has been reported that self-rated health deteriorated consistently with a decrease in sports and exercise over a 4-year observation period among adolescents ${ }^{8}$. On the other hand Rainey et $a{ }^{25}$, found that highly active adolescents rated their health more poorly and point out two possible reasons for that results: active youth may be more prone to injuries and they may be more concerned about their health.

Because many life-style habits are established during childhood and adolescence $^{26}$, physical activity and exercise habits may also be established during these formative years. This is particularly important in girls since they are usually described as less active than boys ${ }^{27}$. Further, different determinants were identified as significant risk factors of childhood obesity between genders, being low activity associated to obesity in girls ${ }^{28}$. Although some studies indicate that higher BMI is associated with poor $\mathrm{SRH}^{10,24}$, our data suggest that this association is different according to gender. It seems that nutritional status, as measured by BMI, is more likely associated with SRH in boys than girls. This finding is of difficult explanation however several reasons may underline this result. For example, it may be related to boys later puberty when compared to girls. In this period is known to occur a rapid growth in body weight and this body changes may influence how adolescents feel about themselves and their health perception ${ }^{14}$. So, it seems imperative to look at this relationship closer in future studies. In addition, gender differences in health complaints are stronger in countries with a low gender development index score ${ }^{29}$, and low socio-economic status is also associated with 
health problems $s^{30}$. This underscores the need to incorporate socio-contextual factors in the study of gender and health. However, in our findings, despite adjusting for SES, the association of PA and SRH still persists. Therefore, the data presented here offer potential information to sustain the importance of PA on overall adolescent's health via the measure of SRH used in this study. Although around 40\% of girls and $35 \%$ of boys exceeded screen time guidelines of $2 \mathrm{~h} /$ day, there was no significant association between TVT and SRH in our sample. Our data do not agree with prior reports of PA and screen time in Canadian adolescents, where both variables were positively and inversely, respectively, associated with health perceptions ${ }^{10}$. One reason for this lack of association might be that time spent watching TV could be more related to more objective indicators of health. Despite this, the measure of TVT could also be a limitation, since it only reflects one type of sedentary behavior that could coexist with relatively high physical activity levels among youth, mitigating the immediate effect on health perception.

There are also some other limitations that need to be considered when interpreting the impact of the data presented here. Firstly, the use of a measure of self-rated health has some limitations as an indicator of "state of health". However, perceptions of health are equally important in regard to health promotion campaigns. Second, the broad age spectrum may be a limitation despite SRH has been shown to be a stable construct over adolescence ${ }^{8,12}$ and our sample is over 13 years' old, biological maturation and the onset of puberty (that varies among individuals) may influence health perception ${ }^{14}$. Finally, our data is from a convenience sample and may not represent the population, therefore limiting the generalizability of our findings. Despite these limitations, this study has extended the research investigating the importance of PA in adolescents' health. The data presented here identifies a potential association between PA and SRH. This, in turn, expresses that higher PA is associated with psychological well-being. BMI was also associated with SRH but only in boys suggesting that it may affect mental health; therefore, gender differences should be considered while designing, adolescent health programs. Public health promotion activities aiming youth should therefore be advocated and may benefit young people's overall health and well-being.

\section{Acknowledgements}

This work was supported by Foundation of Science and Technology - FCT/UID/ DTP/00617/201 and SFRH/BPD/105071/2014.

\section{Conflict of Interest}

The authors declare no conflict of interests.

\section{Contribution of the authors}

J. Mota (0000-0001-7571-9181) and M. Duncan (0000-0002-2016-6580) were responsible for the initial conception, design of the study and gave significant suggestions to the manuscript improvement. M. Barros and J.C. de Farias Júnior (0000-0002-1082-6098) supported data analysis and interpretation and have commented on relevant issues of the manuscript.J.C. Ribeiro (0000-0001-6628-4606), J. Carvalho (0000-0001-6500-7543) and M.P. Santos (0000-0002-2182-9841) have made substantial contributions in data acquisition and added to further development and critical revision of the manuscript. A.N. Pizarro (0000-0001-6518-5569) drafted and coordinated the paper. All authors read and approved the present manuscript. 


\section{References}

1. Telama R, Yang X, Laakso L, Viikari J. Physical activity in childhood and adolescence as predictor of physical activity in young adulthood. Am J Prev Med. 1997;13(4):317-23.

2. Cole TJ, Bellizzi MC, Flegal KM, Dietz WH. Establishing a standard definition for child overweight and obesity worldwide: international survey. BMJ. 2000;320(7244):1240-3.

3. Janssen I, LeBlanc AG. Systematic review of the health benefits of physical activity and fitness in school-aged children and youth. Int J Behav Nutr Phys. 2010;7:40-56.

4. Ortega FB, Ruiz JR, Castillo MJ, Sjostrom M. Physical fitness in childhood and adolescence: a powerful marker of health. Int J Obes. 2008;32(1):1-11.

5. Lobstein T, Frelut ML. Prevalence of overweight among children in Europe. Obes Rev. 2003;4(4):195-200.

6. Sardinha LB, Santos R, Vale S, Silva AM, Ferreira JP, Raimundo AM, et al. Prevalence of overweight and obesity among Portuguese youth: a study in a representative sample of 10-18-year-old children and adolescents. Int J Pediatr Obes. 2011;6(2-2):e124-8.

7. Wade TJ, Pevalin DJ, Vingilis E. Revisiting student self-rated physical health. J Adolesc. 2000;23(6):785-91.

8. Breidablik HJ, Meland E, Lydersen S. Self-rated health during adolescence: stability and predictors of change (Young-HUNT study, Norway). Eur J Public Health. 2009;19(1):73-8.

9. Zullig KJ, Valois RF, Huebner ES, Drane JW. Adolescent health-related quality of life and perceived satisfaction with life. Qual Life Res. 2005;14(6):1573-84.

10. Vingilis ER, Wade TJ, Seeley JS. Predictors of adolescent self-rated health. Analysis of the National Population Health Survey. Can J Public Health. 2002;93(3):193-7.

11. Krause NM, Jay GM. What do global self-rated health items measure? Med Care. 1994;32(9):930-42.

12. Boardman JD. Self-rated health among U.S. adolescents. J Adolescent Health. 2006;38(4):401-8.

13. Mota J, Santos R, Pereira M, Teixeira L, Santos MP. Perceived neighbourhood environmental characteristics and physical activity according to socioeconomic status in adolescent girls. Ann Hum Biol. 2011;38(1):1-6.

14. Currie C, Zanotti C, Morgan A, Currie M, de Looze M, Roberts C, et al. Social determinants of health and well-being among young people: Health Behaviour in School-Aged Children (HBSC) study : international report from the 2009/2010 survey. Copenhagen: WHO Regional Office for Europe; 2012.

15. Tremblay S, Dahinten S, Kohen D. Factors related to adolescents' self-perceived health. Health Rep. 2003;14 Suppl:7-16.

16. Joffer J, Jerdén L, Öhman A, Flacking R. Exploring self-rated health among adolescents: a think-aloud study. BMC Public Health. 2016;16(1):156-66.

17. Wiklund M, Malmgren-Olsson E-B, Öhman A, Bergström E, Fjellman-Wiklund A. Subjective health complaints in older adolescents are related to perceived stress, anxiety and gender - a cross-sectional school study in Northern Sweden. BMC Public Health. 2012;12(1):993-1006.

18. Bailis DS, Segall A, Chipperfield JG. Two views of self-rated general health status. Soc SciMed. 2003;56(2):203-17.

19. Physical Activity Guidelines Advisory Committee. Physical Activity Guidelines Advisory Committee Report, 2008. Washington, DC: US Department of Health and Human Services; 2008.

20. Lubans D, Richards J, Hillman C, Faulkner G, Beauchamp M, Nilsson M, et al. Physical Activity for Cognitive and Mental Health in Youth: A Systematic Review of Mechanisms. Pediatrics. 2016;138(3):piie20161642.

21. Hallal PC, Victora CG, Azevedo MR, Wells JC. Adolescent physical activity and health: a systematic review. Sports Med. 2006;36(12):1019-30.

22. Eime RM, Young JA, Harvey JT, Charity MJ, Payne WR. A systematic review of the psychological and social benefits of participation in sport for children and adolescents: informing development of a conceptual model of health through sport. Int J Behav Nutr Phys Act. 2013;10:98-119.

23. Piko B. Health-related predictors of self-perceived health in a student population: the importance of physical activity. J Community Health. 2000;25(2):125-37. 
24. Page RM, Suwanteerangkul J. Self-rated health, psychosocial functioning, and healthrelated behavior among Thai adolescents. Pediatr Int. 2009;51(1):120-5.

25. Rainey CJ, McKeown RE, Sargent RG, Valois RF. Adolescent Athleticism, Exercise, Body Image, and Dietary Practices. Am J Health Behav.1998;22(3):193-205.

26. Sawyer SM, Afifi RA, Bearinger LH, Blakemore SJ, Dick B, Ezeh AC, et al. Adolescence: a foundation for future health. Lancet. 2012;379(9826):1630-40.

27. Sallis JF, Prochaska JJ, Taylor WC. A review of correlates of physical activity of children and adolescents. Med Sci Sports Exerc. 2000;32(5):963-75.

28. Danielzik S, Czerwinski-Mast M, Langnase K, Dilba B, Muller MJ. Parental overweight, socioeconomic status and high birth weight are the major determinants of overweight and obesity in 5-7 y-old children: baseline data of the Kiel Obesity Prevention Study (KOPS). Int J Obes Relat Metab Disord. 2004;28(11):1494-502.

29. Perneger TV, Gayet-Ageron A, Courvoisier DS, Agoritsas T, Cullati S. Self-rated health: analysis of distances and transitions between response options. Qual Life Res. 2013;22(10):2761-8.

30. Ravens-Sieberer U, Torsheim T, Hetland J, Vollebergh W, Cavallo F, Jericek H, et al. Subjective health, symptom load and quality of life of children and adolescents in Europe. Int J Public Health. 2009;54 Suppl 2:151-9.

\section{Corresponding}

\section{Author}

Andreia Pizarro

andreia.pizarro@gmail.com
Research Centre in Physical Activity,

Health and Leisure

Faculty of Sport, University of Porto

Rua Dr. Plácido Costa, 91, 4200-450

Porto, Portugal

Phone: +351 225074785

Fax: +351225500689
Received

Revised

08/04/2016 $03 / 11 / 2016$ $21 / 12 / 2016$

Approved $\quad 10 / 02 / 2017$ 